\title{
Clinical significance of metallothioneins in cell therapy and nanomedicine
}

This article was published in the following Dove Press journal:

International Journal of Nanomedicine

16 April 2013

Number of times this article has been viewed

\section{Sushil Sharma' \\ Afsha Rais' \\ Ranbir Sandhu' \\ Wynand Nel' \\ Manuchair Ebadi²}

'Saint James School of Medicine,

Bonaire, The Netherlands;

${ }^{2}$ Department of Pharmacology,

Physiology, and Therapeutics, Center of Excellence in Neuroscience, University of North Dakota School of Medicine and Health Sciences,

Grand Forks, ND, USA

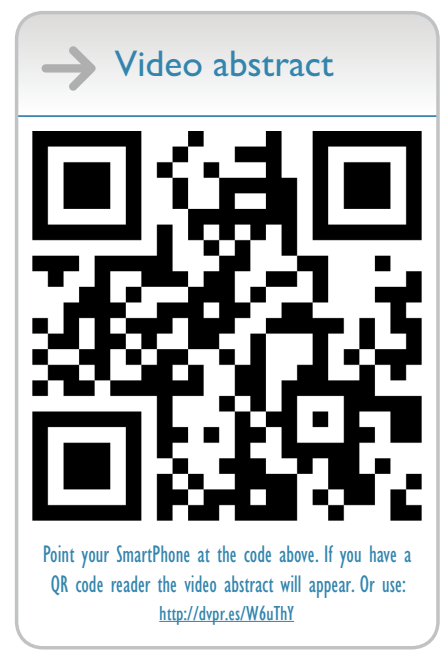

Correspondence: Sushil Sharma Saint James School of Medicine,

Plaza Juliana 4, Kralendijk, Bonaire,

The Netherlands

Tel +3I 5997177550

Fax +3I 5997177570

Email sharma@mail.sjsm.org
Abstract: Mammalian metallothioneins (MTs) are low molecular weight (6-7 kDa) cysteinerich proteins that are specifically induced by metal nanoparticles (NPs). MT induction in cell therapy may provide better protection by serving as antioxidant, anti-inflammatory, antiapoptotic agents, and by augmenting zinc-mediated transcriptional regulation of genes involved in cell proliferation and differentiation. Liposome-encapsulated MT-1 promoter has been used extensively to induce growth hormone or other genes in culture and gene-manipulated animals. MTs are induced as a defensive mechanism in chronic inflammatory conditions including neurodegenerative diseases, cardiovascular diseases, cancer, and infections, hence can serve as early and sensitive biomarkers of environmental safety and effectiveness of newly developed NPs for clinical applications. Microarray analysis has indicated that MTs are significantly induced in drug resistant cancers and during radiation treatment. Nutritional stress and environmental toxins (eg, kainic acid and domoic acid) induce MTs and aggregation of multilamellar electron-dense membrane stacks (Charnoly body) due to mitochondrial degeneration. MTs enhance mitochondrial bioenergetics of reduced nicotinamide adenine dinucleotide-ubiquinone oxidoreductase (complex-1), a rate-limiting enzyme complex involved in the oxidative phosphorylation. Monoamine oxidase-B inhibitors (eg, selegiline) inhibit $\alpha$-synuclein nitration, implicated in Lewy body formation, and inhibit 1-methyl 4-phenylpyridinium and 3-morpholinosydnonimineinduced apoptosis in cultured human dopaminergic neurons and mesencephalic fetal stem cells. MTs as free radical scavengers inhibit Charnoly body formation and neurodegenerative $\alpha$-synucleinopathies, hence Charnoly body formation and $\alpha$-synuclein index may be used as early and sensitive biomarkers to assess NP effectiveness and toxicity to discover better drug delivery and surgical interventions. Furthermore, pharmacological interventions augmenting MTs may facilitate the theranostic potential of NP-labeled cells and other therapeutic agents. These unique characteristics of MTs might be helpful in the synthesis, characterization, and functionalization of emerging NPs for theranostic applications. This report highlights the clinical significance of MTs and their versatility as early, sensitive biomarkers in cell-based therapy and nanomedicine.

Keywords: metallothioneins, free radicals, Charnoly body, $\alpha$-synuclein index, nanomedicine, toxicity, stem cells, theranostics

\section{Introduction}

Mammalian metallothioneins (MT-1-4) are ubiquitous, low molecular weight $(6-7 \mathrm{kDa})$, cysteine-rich, metal-binding proteins existing in all prokaryotes to eukaryotes. MTs were discovered for the first time by Marghoshes and Vallee in horse kidneys, ${ }^{1}$ and in the rodent brain by the authors' group. ${ }^{2}$ Several researchers have elucidated the detailed molecular structure of MTs by performing nuclear magnetic 
resonance spectroscopic analysis. ${ }^{3-7}$ Zangger et al have determined the three-dimensional structure of mouse MT-1 by nuclear magnetic resonance analysis. ${ }^{8}$ Briefly, these polypeptides possess $60-67$ amino acid residues and 20 cysteine molecules which provide antioxidant properties to these metal detoxifying proteins. MTs possess two metal-thiolate clusters (C-terminal $\alpha$-domain and $\mathrm{N}$-terminal $\beta$-domain), including 20 cysteine residues and sulfur atoms, which bind to divalent or monovalent cations. ${ }^{9}$ These domains are linked by a short peptide containing amino acid residue 30-32 in mammalian MTs. In the $\beta$-domain, three divalent or six monovalent metal ions are coordinated, while in the $\alpha$-domain, four divalent or six monovalent cations remain bound. Zinc $(\mathrm{Zn})$ is one of the most abundant divalent metal ions, and its concentration is greater than copper and manganese in the brain. Since free $\mathrm{Zn}$ is a potent inhibitor of sulfhydryl (-SH) enzymes, $\mathrm{Zn}$ in the brain is predominantly MT bound. However, there are several other proteins with which $\mathrm{Zn}$ can bind in the central nervous system (CNS) beyond the scope of this article. Unlike the hepatic or renal $\mathrm{Zn}$ thioneins, the $\mathrm{Zn}$-binding proteins in the brain are not inducible following administration of $\mathrm{Zn}$ as it does not cross the blood-brain barrier efficiently. During oxidative stress, glutathione is significantly reduced. ${ }^{10}$ Nitric oxide-mediated metal release is modulated from MTs by the redox state of glutathione in vitro. ${ }^{11}$ MT induction attenuated carmustine-induced hippocampal toxicity, prevented glutathione reductase inhibition and glutathione depletion, and reduced tumor necrosis factor- $\alpha$, malondialdehyde, and caspase- 3 activity with preservation of cognition in rats. ${ }^{12}$ These findings suggest the therapeutic potential of MTs in neurodegenerative disease and other disorders. Ferric nitrilotriacetate, which produces reactive oxygen species, induced MTs in the liver and kidney. Cadmium (Cd) released after an injection of ferric nitrilotriacetate induced MTs. Thus, MTs may act as antioxidants to compensate for glutathione depletion. MTs are more potent antioxidants compared to glutathione. ${ }^{13}$ Thus, glutathione and MT synthesis is induced as an attempt to combat iron-induced oxidative stress. ${ }^{14}$ Previous studies have shown that MTs are low molecular weight, heat-stable cytosolic proteins with a high content of cysteinyl sulfur that bind heavy metals like $\mathrm{Cd}, \mathrm{Zn}$, and copper. ${ }^{15}$ As these proteins are induced following exposure to heavy metals, it is now well established that they have a detoxifying role during heavy metal toxicity. The primary function of MTs is in the homeostasis of the essential metals $\mathrm{Zn}$ and copper. In addition, a role MT plays in selenium (Se) metabolism in primates has been established. Furthermore, MT has gained significance in the clinical disorders related to trace metal metabolism. This report highlights some of the authors' recent work on MTs and NPs. It also provides a brief update of this emerging field by highlighting the work from other laboratories.

\section{Molecular biology of MTs}

There are primarily four major isoforms of MTs. MT-1 and MT-2 are present ubiquitously; MT-3 is localized predominantly in the hippocampus and its depletion is implicated in the etiopathogenesis of Alzheimer's disease (AD). ${ }^{16}$ Recent studies have shown that MT3 expression is downregulated in the esophageal squamous cell carcinoma by DNA methylation. ${ }^{17}$ MT-4 is localized in the buccal mucosa and pseudostratified squamous epithelial lining of the uterine cavity and is involved in desquamation during menstruation. The exact biological significance of MT-4 is yet to be established. MTs are capable of preventing oxidative stress and apoptotic cell death in the CNS. MTs promote neuronal survival and regeneration in vivo and are protective against metal ion toxicity, oxidative stress, and cytokine injury due to cerebral ischemia or infection, hence could be considered as early and sensitive biomarkers of redox signaling in neurodegenerative disorders such as Parkinson's disease (PD), AD, multiple system atrophy, stroke, and epilepsy. However, the exact molecular mechanism of MT-mediated neuroprotection in these and other neurodegenerative disorders remains elusive. By using MT gene-manipulated mice and aging mitochondrial genome knockout $\left(\mathrm{RhO}_{\text {mgko }}\right)$ cybrids as experimental models of PD and aging and micropositron emission tomography neuroimaging with fluorine18-L-dihydroxyphenylalanine and ${ }^{18} \mathrm{~F}$ fluorodeoxyglucose, it has been established that MTs may provide dopaminergic neuroprotection by (1) augmenting mitochondrial coenzyme $\mathrm{Q}_{10}$ synthesis, (2) attenuating $\alpha$-synuclein nitration, (3) preserving mitochondrial glutathione, (4) enhancing neuromelanin synthesis, (5) preserving ferritin, (6) preventing metal ion accumulation, (7) acting as free radical scavengers, (8) attenuating peroxynitrite $\left(\mathrm{ONOO}^{-}\right)$ion neurotoxicity, (9) maintaining intracellular redox balance, (10) or through all of these mechanisms. ${ }^{18}$ Whether augmentation of coenzyme $\mathrm{Q}_{10}$, glutathione, ferritin, melatonin, and neuromelanin synthesis in MT transgenic mice CNS occurs independently, is dependent on each other, or occurs synergistically, remains unknown. ${ }^{19}$ Although it has been discovered that 3-morpholinosydnonimine (or SIN-1: a potent peroxynitrite donor) and 1-methyl, 4-phenyl, 1,2,3,6-tetrahydropyridineinduced $\alpha$-synuclein nitration is attenuated in MT transgenic mice striatum, knowledge is very limited regarding the exact 
functional significance of these findings. In a recent study, the neuroprotective role of MTs in 3-morpholinosydnonimine and 1-methyl, 4-phenyl, 1,2,3,6-tetrahydropyridine-induced oxidative and nitrative stress was investigated, with a primary objective to explain the basic molecular mechanism of MTmediated neuroprotection in PD and other neurodegenerative disorders. Based on these findings, it was reported that MTs are capable of inhibiting $\alpha$-synuclein index and broadly classified neurodegenerative $\alpha$-synucleinopathies. ${ }^{20-23} \mathrm{Cd} / \mathrm{Se}$ NP-labeled bone marrow-derived stem cells have also been used to determine their biodistribution, pharmacokinetics, and therapeutic potential in an experimental model of acute ischemic stroke (AIS) and have suggested that the therapeutic potential of stem cells can be augmented by MT induction. ${ }^{24,25}$ Furthermore, it was proposed that MT transgenic striatal fetal stem cells can be implanted in the striatal region of the homozygous weaver mutant (wv/wv) mice exhibiting progressive neurodegeneration and typical symptoms of $\mathrm{PD}$, $\mathrm{AD}$, and drug addiction to evaluate the therapeutic potential of MTs. ${ }^{26}$

\section{MTs and Zn homeostasis}

$\mathrm{Zn}$ dyshomeostasis has been recognized as an important mechanism of cell death in acute brain injury. An increase in the level of free or histochemically reactive $\mathrm{Zn}$ in astrocytes and neurons is considered one of the major causes of cell death in ischemia and trauma. Although Zn dyshomeostasis can lead to cell death via diverse routes, the major pathway appears to involve oxidative stress. Recently, it has been discovered that a rise of $\mathrm{Zn}$ in autophagic vacuoles, including autolysosomes, is a prerequisite for lysosomal membrane permeabilization and cell death in cultured neurons exposed to oxidative stress. The source of $\mathrm{Zn}$ in this process is redoxsensitive Zn-binding protein MTs, which release Zn during oxidative stress. Of the MTs, MT-3 is particularly enriched in the CNS, but its exact biological significance is yet to be established. Like other MTs, MT-3 may function as a metal detoxicant, but may also inhibit neurite outgrowth and promote neuronal death by serving as a source of toxic $\mathrm{Zn}$ release. In addition, MT-3 regulates lysosomal functions. In the absence of MT-3, there are changes in lysosomeassociated membrane protein- 1 and -2 and reductions in lysosomal enzymes that result in decreased autophagic flux, which may have dual effects on cell survival. In acute oxidative injury, $\mathrm{Zn}$ dyshomeostasis and lysosomal membrane permeabilization are diminished in MT-3 knockout cells, resulting in reduced cell death. But during the chronic phase, diminished lysosomal function may lead to the accumulation of abnormal proteins and cytotoxicity. The role of $\mathrm{Zn}$ and MT-3 in autophagy and/or lysosomal function is now being explored. In light of evidence that autophagy and lysosomes may play significant roles in the pathogenesis of various neurological diseases, further insight into the contribution of $\mathrm{Zn}$ dynamics and MT-3 function may provide avenues to effectively regulate these processes in the $\mathrm{CNS}^{27} \mathrm{It}$ has been discovered that the incidence of lysosomes and Charnoly body (CB) formation is significantly increased during nutritional stress and during environmental insult including kainic acid and domoic acid neurotoxicity, which may enhance MTs expression as a defensive mechanism (Figure 1A). CBs are electron-dense, multilamellar stacks of degenerated mitochondrial membranes that are generated as a consequence of free radical overproduction during oxidative and nitrative stress. MTs inhibit CB formation by acting as free radical scavengers to provide mitochondrial protection. Unfortunately, increased lysosomal activity as a consequence of severe nutritional stress or environmental

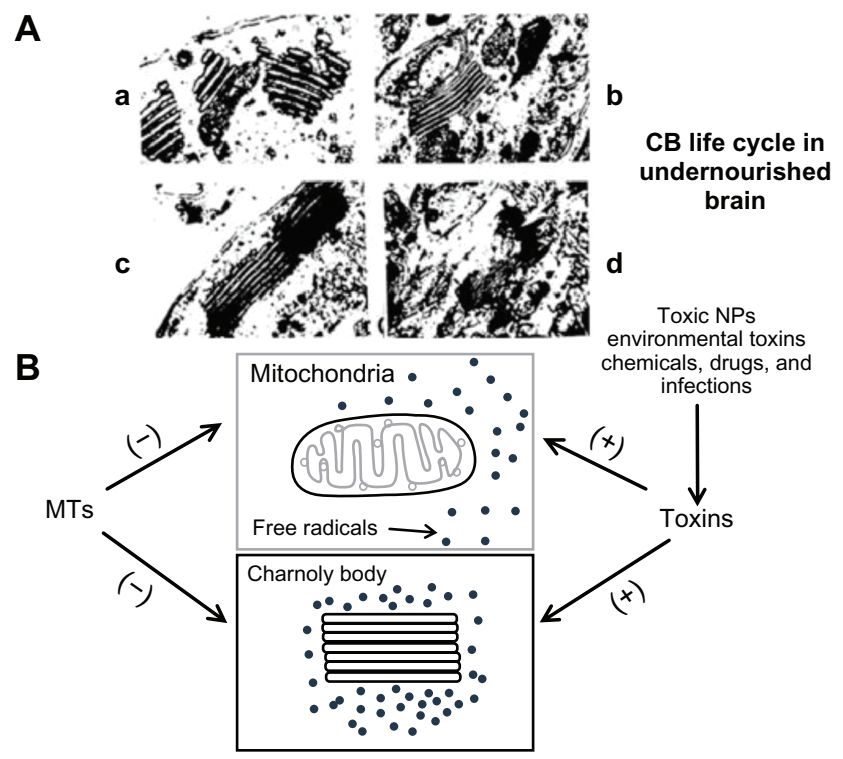

Figure I (A) Transmission electron microscope (magnification 50,000x) image illustrating $\mathrm{CB}$ formation in the developing undernourished Purkinje neurons as a consequence of degeneration of mitochondrial membranes (a). These membranes are transformed in to multilamellar (usually pentalamellar or heptalamellar) electrondense structures (b) to form mature CBs (c). The structural degradation of CBs occurs eventually due to increased lysosomal activity (d). Based on the transmission electron microscopy and electrophysiological studies, it is proposed that CBs are involved in desmosomal repair, autophagy, and increased afterhyperpolarization duration due to increased intracellular calcium ions $\left(\mathrm{Ca}^{2+}\right)$ in the 15 -day undernourished rat cerebellar Purkinje neurons. (B) Free radicals (indicated by dots) are generated as a byproduct of mitochondrial oxidative phosphorylation.

Notes: Toxic NPs, environmental toxins, chemicals, drugs, nutritional stress, and infections augment free radical overproduction, which may trigger $C B$ formation. MTs serve as free radical scavengers to protect mitochondrial structural and functional integrity by inhibiting $C B$ formation. $C B$ formation is a transitory state between apoptosis and cell death and is a reversible process. $(+)$ indicates activation; $(-)$ indicates inhibition.

Abbreviations: $C B$, Charnoly body; MT, metallothionein; NP, nanoparticle. 
neurotoxicity may structurally degrade induced MTs as well, thus compromising the intracellular defensive mechanisms which may lead to early morbidity and mortality in progressive neurodegenerative disorders including PD, AD, and drug addiction. Similarly, toxic NPs may induce autophagy as a result of intracellular acidosis which may trigger CB formation and its degradation by lysosomal activation during nutritional stress and/or environmental toxicity (Figure 1B). Recently, a peptide termed EmtinB has been designed, which is modeled after the $\beta$-domain of MT- 2 and mimics the biological effects of MT-1/2 in vitro. ${ }^{28}$ The neuroprotective effect of EmtinB in the in vitro and in vivo models of kainic acid-induced neurotoxicity has been investigated. EmtinB can pass through blood-brain barrier and is detectable in the plasma for up to 24 hours. Indeed, treatment with EmtinB attenuated seizures in $\mathrm{C} 57 \mathrm{BL} / 6 \mathrm{~J}$ mice exposed to moderate $(20 \mathrm{mg} / \mathrm{kg})$ and high $(30 \mathrm{mg} / \mathrm{kg})$ kainic acid doses and decreased mortality. Furthermore, EmtinB treatment reduced kainic acid-induced neurodegeneration in the CA1 region, suggesting its role as a target for therapeutic development.

\section{MTs attenuate domoic acid toxicity}

Domoic acid is an excitatory amino acid and a rigid structural analog to glutamate and kainic acid which acts through glutamate receptors and elicits a rapid and potent neurotoxic response. Domoic acid continues to pose a global risk to the health and safety of humans and wildlife. It is a marine biotoxin associated with harmful algal blooms and is the causative agent of amnesic shellfish poisoning in marine animals and humans. The hippocampus is a specific target site having increased sensitivity to domoic acid toxicity. It has been discovered that even intrauterine exposure to domoic acid can induce damage to hippocampal CA3 and dentate nucleus in the progeny as noticed in AD patients with no overt clinical seizure activity. ${ }^{29}$ Histopathological evidence indicates that in addition to neurons, the astrocytes are also injured as consequence of domoic acid toxicity. Furthermore, the effect of domoic acid has been confirmed in cultured primary astrocytes from the hippocampus and the brain stem. The biomarker analysis for the early response genes including c-Fos, c-Jun, c-Myc, Hsp-72, specific marker for the astrocytes - glial fibrillary acidic protein - and the glutamate receptors including glutamate receptor-2, N-methylD-aspartic acid receptor-1, -2A, and -2B has been performed by microarray analysis. ${ }^{30}$ Although, the astrocyte glial fibrillary acidic protein and c-Fos were not affected, c-Jun and glutamate receptor-2 were downregulated. The chemokines/ cytokines, tyrosine kinases (Trk), and apoptotic genes were also altered. The cytokines that were upregulated included interleukin- $1 \alpha$ (IL- $1 \alpha)$, IL-1 $\beta$, IL-6, the small inducible cytokine, interferon protein 10P-10, CXC chemokine LIX, and insulin-like growth factor-binding proteins, whereas Bax, Bcl-2, Trk-A, and Trk-B were downregulated. Only the hippocampal astrocytes were affected, suggesting that astrocytes may be used as a pharmacological target for the prevention and treatment of domoic acid poisoning and for other CNS pathologies involving excitotoxicity. A comprehensive review of domoic acid-induced brain pathology including ultrastructural changes associated to subchronic oral exposure, its molecular mechanism of aggregation in NPs, cell/tissue injury, food safety, and human health issues is now available. ${ }^{31}$ Recently, field studies in the Pacific Ocean and laboratory studies have detected increased domoic acid production under conditions of iron limitation. ${ }^{32}$

\section{MTs and stem cells in experimental autoimmune encephalomyelitis (EAE)}

MTs as anti-inflammatory and neuroprotective macromolecules are induced during EAE and multiple sclerosis. ${ }^{33} \mathrm{EAE}$ and multiple sclerosis are characterized by significant inflammation, demyelination, neuroglial damage, and cell death. EAE is characterized by demyelination, inflammation, and neurodegeneration of CNS, in which free radicals play a significant role. Exogenous administration of Zn-bound MT-2 to Lewis rats with EAE reduced clinical symptoms and the inflammatory response, oxidative stress, and apoptosis of the CNS areas. Zn-bound MT-2 treatment prevented demyelination and axonal damage and transection, and stimulated oligodendroglial regeneration, as well as the expression of the basic fibroblast growth factor, transforming growth factor- $\beta$, neurotrophin-3, neurotrophin- $4 / 5$, and nerve growth factor. These beneficial effects of Zn-bound MT-2 could not be attributable to its $\mathrm{Zn}$ content, suggesting potential application of Zn-bound MT-2 as a safe and successful therapy for multiple sclerosis. Recently, the efficacy of murine mesenchymal stem cells (MSCs) as treatment of EAE induced in mice by the encephalitogenic peptide myelin oligodendrocyte glycoprotein (35-55) has been demonstrated. ${ }^{34}$ Various biomarkers of oxidative stress, inflammation/degeneration, and apoptosis such as MTs, antioxidant enzymes (superoxide dismutase, catalase, and glutathione-S-transferase), poly(adenosine diphosphate ribose) polymerase-1, and p53 during EAE progression and following MSC treatment have been analyzed. MT expression was significantly increased in EAE mice compared with healthy controls, but while 
expression of MT-1 and MT-3 increased along EAE course, MT-2 was upregulated at the onset, but returned to control levels during chronic phase. The changes in the transcription and activity of the antioxidant enzymes and poly(adenosine diphosphate ribose) polymerase-1 and p53 expressions exhibited similar kinetics for MT-1 and MT-3. MSCs reduced the EAE-induced increases in activities of all these proteins supporting an antioxidant and neuroprotective role of MSCs that was further confirmed in vitro in neuroblastoma cells exposed to oxidative stress. ${ }^{35}$

\section{MTs and liposome-encapsulated stem cells}

MSC transplantation is now a promising method which is being actively explored in regenerative medicine. Thus, gene-modified MSCs (such as MTs) may possess superior characteristics of specific tissue differentiation, resistance to apoptosis, and directional migration. However, viral vectors have the disadvantages of potential immunogenicity, carcinogenicity, and complicated synthetic procedures. Polyethylene glycol (PEG)-grafted polyethyleneimine holds promise in gene delivery because of easy preparation and targeting modification. Coaxial electrospinning enables the incorporation of liposomes into nanofibers, and polyvinyl alcohol core/poly- $\varepsilon$-caprolactone shell nanofibers with embedded liposomes can preserve the enzymatic activity of encapsulated horseradish peroxidase. The clinical potential of this system has been demonstrated by the enhancement of MSC proliferation, suggesting that intact liposomes incorporated into nanofibers by coaxial electrospinning may serve as promising drug delivery vectors. ${ }^{36}$

Recently, investigators have synthesized a PEG 8000-grafted polyethylenimine 25,000 graft copolymer and used agarose gel retardation assay and dynamic light scattering to determine the properties of NPs. ${ }^{37} \mathrm{~A}$ multifunctional envelope-type nano device (MEND) has been developed as a novel nonviral gene delivery system. ${ }^{38}$ Furthermore, a study has been performed to determine the effect of systemic delivery of prednisolone phosphate encapsulated within "stealth" liposomes on bone erosion and osteoclast activity during experimental antigen-induced arthritis. ${ }^{39}$ As preclinical evaluation in animals does not necessarily portray human responses, liposome-encapsulated hemoglobin, an artificial oxygen carrier, has been tested in immunodeficient mice reconstituted with human hematopoietic stem cells (cord blood-transfused NOD/SCID/IL-2R $\gamma$ null) mice. ${ }^{40}$ In a further study, investigators have attempted to improve the specificity and efficiency of gene transfection and make the liposome a better gene transfer vector to the brain by using monoclonal antibody (anti-Lex/stage-specific embryonic antigen-1)-mediated targeting of liposomes. ${ }^{41}$ To determine the effect on erythropoiesis of liposome-encapsulated dichloromethylene diphosphonate-induced changes in bone marrow macrophages, red blood cell parameters and the formation of erythroid burst-forming unit-derived colonies in vitro have been evaluated. ${ }^{42}$ It is known that the human multidrug resistance (MDRl) gene encodes a $170 \mathrm{kD}$ glycoprotein (P-glycoprotein), which is an adenosine triphosphate-dependent transmembrane efflux pump for many different cytotoxic drugs. Therefore, an efficient expression of the human $M D R 1$ gene in mouse bone marrow cells after transfection with a liposomal delivery system has been developed. ${ }^{43}$ In another study, investigators compared the toxic effects of actinomycin-D encapsulated either in the aqueous phase or in the lipid phase of liposomes and the nonencapsulated actinomycin-D on the blood forming system on cell proliferation in the intestine and on antibody production by spleen lymphocytes and have confirmed that actinomycin$\mathrm{D}$ is less toxic to mice than nonencapsulated actinomycin-D, but retains its tumoricidal activity. ${ }^{44}$ Similarly, nerve growth factor has also been encapsulated into liposomes in order to protect it from the enzyme degradation in vivo and promote permeability across the blood-brain barrier. ${ }^{45}$ Furthermore, MEND has been developed as a novel nonviral gene delivery system. ${ }^{46}$ The host response to systemically administered lipid NPs encapsulating plasmid DNA has been studied in the spleen using a DNA microarray and MEND. ${ }^{47}$ These authors have established that PEGylation is a useful method for achieving a longer circulation time for delivery of MEND to a tumor via the enhanced permeability and retention effect. However, PEGylation inhibits cellular uptake and endosomal escape, which results in significant loss of activity for the delivery system. Therefore, for successful gene delivery, particularly for cancer treatment, the crucial issue associated with the use of PEG - the "PEG dilemma" - has to be addressed. As many as 1581 differentially expressed genes have been identified by PEG-unmodified NPs. PEGylation of NPs caused reduction in the expression of most of the genes. However, type I interferon expression was significantly increased. Based on these studies, it has been hypothesized that PEGylation inhibits the endosomal escape of NP and extends the interaction of toll-like receptor-9 with CpG DNA accompanied by the production of type I interferon. This hypothesis has been tested by introducing a $\mathrm{pH}$-sensitive fusogenic peptide, GALA, which enhances the endosomal escape of PEGylated NP. As expected, type I interferon 
was reduced and IL-6 remained unchanged, suggesting that microarray analysis and the manipulation of intracellular trafficking constitute a rational strategy for reducing the host immune response to NPs. Furthermore, investigators have developed MEND for efficient delivery of nucleic acids. For tumor delivery of MEND, PEGylation has been considered a useful method, which confers a longer systemic circulation and tumor accumulation via the enhanced permeability and retention effect. ${ }^{48}$ As preclinical evaluation in animals does not necessarily portray human responses, liposome-encapsulated hemoglobin (an artificial oxygen carrier) has been tested in immunodeficient (cord bloodtransfused NOD/SCID/IL-2R $\gamma$ null) mice reconstituted with human hematopoietic stem cells. ${ }^{49}$ However, PEGylation inhibits cellular uptake and subsequent endosomal escape. To overcome this, investigators developed a PEG-peptide1,2-dioleoyl-sn-glycero-3-phosphoethanolamine that is cleaved in a matrix metalloproteinase-rich environment. Furthermore, the systemic delivery of small interfering ribonucleic acid to tumors has been examined by employing a MEND that is modified with PEG-peptide-1,2-dioleoyl-snglycero-3-phosphoethanolamine. PEG-peptide-1,2-dioleoylsn-glycero-3-phosphoethanolamine modification accelerated both cellular uptake and endosomal escape, compared to a conventional PEG-modified MEND. It is known that cancers often overexpress epidermal growth factor (EGF) and other growth factors to promote cell replication and migration. Liposomes bearing EGF receptors covalently crosslinked to p-Toluic acid or methyl-PEG 4-N-hydroxysuccinimide ester (ie, modified EGF receptor-bearing liposomes) exhibit an increased rate of release of encapsulated drug compounds when EGF is present in solution. ${ }^{50}$ In all the liposomal encapsulation studies described above, the precise role of MTs has not been explored as yet. Studies in this direction will not only further elucidate the therapeutic potential of liposome-encapsulated genes or drugs but also their safety aspects of NPs for clinical applications.

\section{MTs and NPs}

It is now well established that the emission spectra of surfactant-coated semiconductor NPs depend on the size and the coating material. ${ }^{51-53}$ The blue series represents different sizes of $\mathrm{Cd} / \mathrm{Se} \mathrm{NPs}$ with diameters of 2.1, 2.4, 3.1, 3.6 , and $4.6 \mathrm{~nm}$. The green series is of indium phosphide NPs with diameters of $3.0,3.5$, and $4.6 \mathrm{~nm}$. The red series is of indium arsenide NPs with diameters of 2.8, 3.6, 4.6, and $6.0 \mathrm{~nm}$. A true-color image of a series of silica-coated NPs probes with a $\mathrm{Cd} / \mathrm{Se}$ core and a $\mathrm{Zn}$ sulfide or $\mathrm{Cd}$ sulfide shell in aqueous buffer have also been developed. These NPs can be illuminated with ultraviolet light and used effectively to induce MTs in cell-based therapies as discussed in this report. Recently, $\mathrm{Cd} / \mathrm{Se} /$ tellurium (Te)-based quantum dots (QD-705) have been developed with potential for biomedical applications. Although the biological fate of QD-705 is established, its chemical fate in the biological system still remains uncertain. One study investigated the chemical fate of QD-705 in mice kidneys. The molar ratio of $\mathrm{Cd}$ and $\mathrm{Te}$ (increased $\mathrm{Cd} / \mathrm{Te}$ ratio signifies increased $\mathrm{Cd}$ release from QD-705) and the induction of tissue MTs are markers for elevated free Cd in tissues. Almost 100\% of QD-705 was retained in the body even 16 weeks after exposure, with significant pharmacokinetics in the kidneys. Elevations in the $\mathrm{Cd} / \mathrm{Te}$ ratio and MT-1 expression in the kidneys suggested that free $\mathrm{Cd}$ is released from QD-705. Thus, QD-705 is not stable or biologically inert as free $\mathrm{Cd}$ released from QD-705 may increase the risk of nephrotoxicity because MTs bind these metal NPs and are specifically induced to prevent nephrotoxicity. ${ }^{54}$

\section{MTs as molecular magnets}

Recently, novel methods have been developed for preparing molecular magnets and patterning of the molecules on a semiconductor surface. ${ }^{55} \mathrm{~A}$ magnetically aligned MT containing manganese and $\mathrm{Cd}$ is first synthesized, and the molecules are then placed into nanoporous silicon surfaces using electron beam lithography and reactive ionetching techniques. The self-assembled growth of the MT molecules on the patterned silicon surface grows into rodor ring-type three-dimensional nanostructures depending on the patterned surface nanostructures. Scanning electron microscopy, atomic force microscopy, and magnetic force microscopic analyses has revealed that these nanostructures exhibit molecular magnetization and are compatible with conventional semiconductors, which makes manganese and Cd-containing MT-2 NPs candidates for clinical applications and sensing nanodevices.

\section{MTs as early and sensitive biomarkers of NPs toxicity}

There is now evidence that MTs are induced in response to various semiconductor NPs. In particular, silver (Ag) NPs, $\mathrm{Cd}$ sulfide, $\mathrm{Cd} / \mathrm{Te} \mathrm{NPs}$, and carbon nanotubes are known to induce MTs as a defensive mechanism to prevent toxicity. Uncoated $\mathrm{Cd} / \mathrm{Te} \mathrm{QD}$ in freshwater leads to the formation of aggregates and a dissolved component of $\mathrm{Cd}$, where the latter explains the contribution of the accumulation pattern 
in mussel tissues and induced MT levels in mussels. ${ }^{56-58}$ Recently, Neupane has developed MT-capped Cd/Se NPs, in which up to eight MTs could be attached as detected by an increase in the fluorescence intensity. ${ }^{59} \mathrm{Cd} / \mathrm{Se}$ QD NP-labeled bone marrow-derived mononuclear stem cells (MNCs) have been used to determine their biodistribution, pharmacokinetics, and therapeutic potential in experimental model of AIS. ${ }^{60}$ By employing digital fluorescence microscopy and confocal microscopic analysis, it was discovered that MNCs exhibit preferential chemotaxis and are exponentially eliminated from the peri-infarct region as a function of time. These findings led to determining the therapeutic window of MNC-mediated recovery following AIS ${ }^{61}$ A detailed description of cell-based therapy in AIS has also been provided. ${ }^{62}$ Furthermore, it was discovered for the first time that bone marrow-derived MNCs protect cortical neurons by modulating microglia in a cell culture model of AIS; additionally, the basic molecular mechanism of neuroprotection afforded by MNCs was elucidated. ${ }^{63}$ Treatment of MNCs in AIS rats significantly increased brain regional IL-10, hence it is proposed that cortical neurons are protected directly by the MNC-mediated paracrine release of IL-10. Indeed, IL-10 can directly bind to its specific IL-10 receptor to execute anti-inflammatory response by activating upstream phosphatidylinositol 3-kinase and downstream signal transducer and activator of transcription-3-mediated signal transduction cascade. ${ }^{64}$ To further confirm these findings, the effect of transcatheter injections on the viability and cytokine release of mononuclear cells has been examined. ${ }^{65}$ These findings have indicated that MNCs are highly primitive, fragile, and can release anti-inflammatory cytokines such as IL-10 and other neurotrophic factors readily to exert their therapeutic effect in AIS and other neurodegenerative diseases. There could be several other molecular mechanisms in addition to MT-mediated MNC neuroprotection which remain unexplored yet. However, in vivo molecular imaging studies from the authors' laboratories employing ${ }^{18} \mathrm{~F}$ fluorodeoxyglucose and fluorine-18-L-dihydroxyphenylalanine as positron emission tomography biomarkers strongly support MTs as potent antioxidant neuroprotective factors in the progressive neurodegenerative disorders such as $\mathrm{PD}, \mathrm{AD}$, and drug addiction. ${ }^{66}$ Based on these findings, there will be further investigation into the detailed pharmacological properties of NPs using MTs and CB formation as sensitive biomarkers (Figure 2). There is now evidence that Ag NP-induced changes in the action potential are associated with inhibitory effects on voltage-gated sodium currents of hippocampal CA1 neurons. ${ }^{67}$ Furthermore, in vitro studies have indicated that Ag NPs can induce oxidative stress and acute calcium responses in the primary mixed neural cell cultures. ${ }^{68}$ MTs can attenuate these deleterious events by acting as potent antioxidants and anti-inflammatory agents, as previously discussed. ${ }^{25,26}$ Further studies in this direction will not only enhance the theranostic potential but also the safety measure of NPs for future biomedical applications.

\section{MTs for future NPs development}

Oxidative stress and secondary damage can occur during carcinogenesis and neurodegenerative disorders, as has been reported recently. ${ }^{25}$ MTs as reactive oxygen species scavengers inhibit oxidative stress due to their metal ion release and inhibit apoptosis by attenuating mitochondrial cytochrome c leakage and caspase-3 activation. It is well established that MTs are also significantly induced by metal NPs and by environmental neurotoxins. To test the prolonged consequences of a short transient exposure of astrocytes to Ag NPs, cultured primary astrocytes were incubated for 4 hours in the presence of Ag NPs, and the cell viability and metabolic parameters were investigated. ${ }^{69} \mathrm{Ag}$ NPs caused concentration-dependent increase in Ag up to $46 \mathrm{nmol} / \mathrm{mg}$ protein, but did not compromise cell viability. During subsequent incubation in Ag NP-free medium, the Ag content remained constant for up to 7 days. The presence of Ag NPs neither induced any delayed cellular toxicity nor glucose metabolism, lactate production, or the ratio of glutathione to glutathione disulfide. However Ag NP-treated astrocytes upregulated MT expression suggesting that MT induction may prevent Ag NP-mediated Ag ion toxicity.

It is now realized that gold ( $\mathrm{Au}$ ) NPs may have theranostic applications in humans. Since Au NPs have significant clinical applications, their release in the environment may induce toxicological effects. In a recent study, the marine bivalve Scrobicularia plana was exposed to $5 \mathrm{~nm}, 15 \mathrm{~nm}$, and $40 \mathrm{~nm}$ Au NPs during a 16-day laboratory exposure at $100 \mu \mathrm{g} \mathrm{L}^{-1} \mathrm{Au} .^{70}$ After exposure to Au NPs forming aggregates $(>700 \mathrm{~nm})$, the $\mathrm{Au}$ was accumulated in the soft tissues. Au NP-induced MTs (5 nm and $40 \mathrm{~nm}$ ) increased activities of catalase and superoxide dismutase and of glutathione S-transferase, indicating induction of the defense mechanisms against NP-induced oxidative stress. Exposure to $\mathrm{Au} \mathrm{NPs}$ also impaired burrowing behavior. However, these effects were observed at higher doses than expected in the environment. Recently, an extended fish embryo test was utilized to bridge the gap between cell culture and small animal models to investigate the toxicity of Au NPs in wild type and transgenic zebrafish. ${ }^{71}$ The fish embryo test con- 


\section{MTs inhibit SI and CBs formation to provide therapeutic effect}

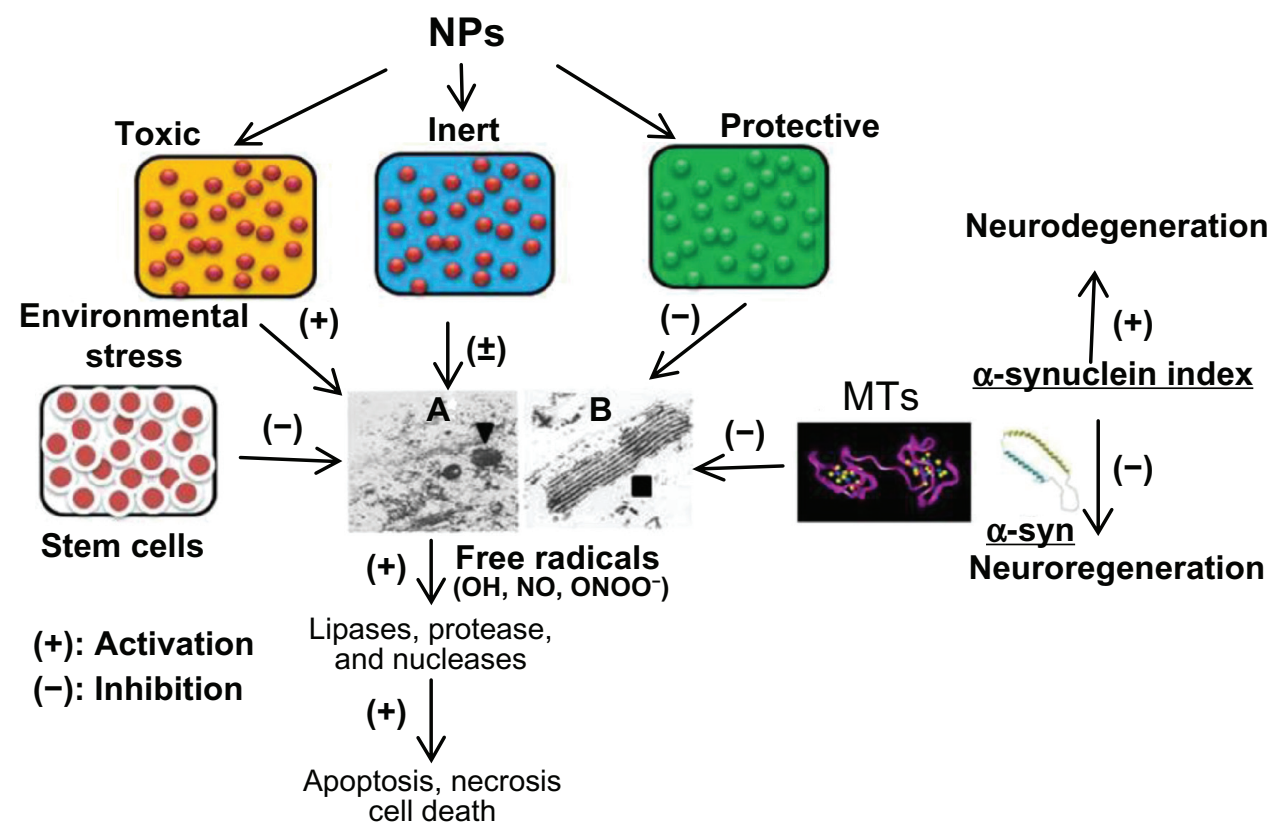

Figure 2 Three types of NPs have been proposed (I) toxic, (2) protective, and (3) inert. NPs augmenting the zinc ion homeostasis could be neuroprotective, those inducing dyshomeostasis could be toxic, and those producing no significant change could be neutral or inert. Thus, metal ion speciation of MTs following NP exposure employing sensitive procedure such as inductively coupled plasma mass spectroscopy may provide a better understanding of the therapeutic potential of MTs in nanomedicine. At the ultrastructural level, protective NPs may prevent CB formation and toxic NPs will induce CB formation, whereas inert NPs will remain ineffective. Stem cells provide MT-mediated mitochondrial protection and inhibit CB formation to facilitate regenerative process. Any physical, physiological, and/or pharmacological intervention involving MT induction will inhibit CB formation and provide neuroprotection. Thus, MT induction and CB formation may be utilized as early and sensitive biomarkers of NPs safety, effectiveness, and toxicity for theranostic applications. (The specificity of these biomarkers may be explored in the future). Furthermore, toxic NPs and environmental stress may induce overproduction of free radicals ( $\mathrm{OH}, \mathrm{NO})$ to cause lysosomal membrane destabilization and $\mathrm{CB}$ formation. Free radicals may induce lipases, proteases, and nucleases to cause membranolysis, proteolysis, and nuclear DNA depolymerization leading to apoptosis, necrosis, and even cell death. MTs may serve as free radical scavengers to inhibit CB formation and provide therapeutic effect in neurodegenerative disease and other disorders. (A) Transmission electron microscope picture (magnification 20,000 $\times$ ) demonstrating the cytoplasmic organization of Purkinje neuron from the undernourished rat cerebellar cortex demonstrating increased incidence of lysosomes ( $\mathbf{)}$ ). (B) Multilamellar electron-dense membrane stacks (CB - ) in the I5-day undernourished rat Purkinje neurons (magnification 50,000X).

Notes: $(+)$ indicates activation; $(-)$ indicates inhibition.

Abbreviations: $\mathrm{CB}$, Charnoly body; MT, metallothionein; NO, nitric oxide; NP, nanoparticle; OH, hydroxyl; ONOO, peroxynitrite; SI, $\alpha$-synuclein index.

firmed all findings of a study in HeLa cells and added new information on teratogenicity and hepatotoxicity that could not be obtained from cultured cells. Recently, mesoporous silicon nanochips have been developed for diagnostic applications. ${ }^{72}$ Biocompatible and biodegradable silicon NPs, mesoporous silicon nanochips, and silicon dioxide nanocoating microdevices can be used for the sensitive and early detection of disease biomarkers from serum using mass spectrometry. Silicon is converted to silicic acid and excreted through the urine. However, if inhaled, it can cause silicosis similar to asbestosis, mesothelioma, and lung cancer. ${ }^{73}$ Although silicon nullifies aluminum toxicity, in the absence of ascorbic acid it may facilitate iron accumulation to induce further toxicity.

Indeed, MTs are protective when induced at the physiological and pharmacological level; however, their enhanced induction may reflect the toxicity of NPs. Particularly when the protective layer of $\mathrm{Zn}$ oxide is removed from QDs in vivo, as discussed above. Recently developed QDs are composed of $\mathrm{Cd} / \mathrm{Se}$, which provides excellent fluorescent properties and may be used for tumor localization to help the surgeon to identify normal cancer cells from cancer stem cells. However, when the protective $\mathrm{Zn}$ oxide layer is dislodged, these NPs could induce $\mathrm{Cd}$ nephrotoxicity. Therefore, the primary objective of coating the NPs with Zn oxide is to avoid direct toxicity and simultaneously induce MTs as protective antioxidants and free radical scavengers to provide mitochondrial protection. Indeed, a considerable amount of research is required in this direction to stabilize NPs in the biological system and enhance their theranostic potential by employing MTs as early and sensitive biomarkers, as emphasized in this report. At present, the exact pathophysiological significance of MT induction is not understood due to a lack of existing knowledge. $\mathrm{Zn}$ ions $\left(\mathrm{Zn}^{2+}\right)$ act as physiological neuromodulators at glutamatergic synapses; however, in order to avoid neurotoxic damage, the intracellular $\mathrm{Zn}^{2+}$ must be controlled by: (1) $\mathrm{Zn}^{2+}$ transporters, (2) $\mathrm{Zn}^{2+}$ 
buffering MTs, and (3) mitochondrial sequestration systems. Particularly in physiological aging, if any of these systems is impaired and/or not adequately coordinated with the other two, the resulting rise of intracellular $\mathrm{Zn}^{2+}$ may inhibit the cellular energy and affect mitochondria as a primary target. ${ }^{74}$ MTs could be directly or indirectly involved in the modulation of cellular senescence and might represent a potential therapeutic target against the aggregation of dysfunctional aging cells. ${ }^{75}$ Therefore, it may be important to perform metal ion speciation of MTs employing atomic absorption spectrophotometry or preferably inductively coupled plasma mass spectrometry to assess their exact pathophysiological significance in neurodegenerative disease and other disorders. For instance, Zn-bound MTs may play a different role during cancer or aging, switching from a protective to a deleterious one in immune, endocrine, and cerebral activities. Intracellular $\mathrm{Zn}^{2+}$ homeostasis is affected by oxidative stress, which is a trigger for detrimental $\mathrm{Zn}^{2+}$ release from MTs. Moreover, $\mathrm{Zn}^{2+}$ at higher concentrations may induce oxidative stress by promoting mitochondrial and extramitochondrial production of reactive oxygen species in the aging brain. Thus, it will be extremely necessary to consider MTs as important biomarkers while developing biologically-safe metal NPs for theranostic applications in future.

\section{Clinical significance of MTs in nanomedicine}

Recent studies have established that bone marrow-derived stem cells are able to alleviate symptoms of lung silicosis. ${ }^{9}$ Thus, MT semiconductor NPs are now being developed to encapsulate silicon as third-generation NPs for theranostic applications. Indeed, semiconductor NPs have potential applications in medicine, engineering, and biology. Size tunable emission, through the quantum confinement effect of semiconductor NPs, make them useful chromophores as fluorescence biomarkers, light emitting diodes, and laser materials. The NPs can be stabilized by thiol-containing capping molecules such as MTs. Although hydrophilic monothiols render the NPs water soluble, they are degraded by light exposure. Thus, peptides based on polycysteinecontaining MTs may provide robust, water soluble, and biocompatible capping groups for developing semiconductor NPs. Up to eight MT peptides may be attached to each $\mathrm{Cd} / \mathrm{Se}$ NPs to provide structural and functional stability and prevent cytotoxicity. ${ }^{76,77}$ Complementary DNA and protein microarray analysis have revealed that various stress-related genes including metallothioneins (MTs), heat shock proteins (HSPs), glutathione S-transferase (GST), p53, cytochrome
p450-1A (Cyp-1A), and transferrin are induced following toxic insult. ${ }^{78}$ MTs are specifically induced in metal toxicity, cancer, inflammation, and infections, hence can serve as early and sensitive biomarkers of environmental safety and effectiveness of newly developed NPs. ${ }^{78,79}$ Because of their unique physical, optical, and mechanical properties, NPs hold great promise in improving clinical diagnosis and effective treatment. Their use in research and consumer products is increasing rapidly, hence contamination of the environment with various NPs seems inevitable. Because surface waters receive pollutants and contaminants from many sources including NPs, and act as reservoirs and conduits for many environmental contaminants, understanding the impacts of NPs on the organisms within these environments is critical for evaluating their toxicity. Thus, MTs could be used as early and sensitive biomarkers to evaluate environmental toxicity. MTs can also serve as early and sensitive biomarkers of neurotoxicity as well as drug addiction. ${ }^{25,26}$ Moreover, frequent structural heterogeneity and mutations in bacterial MTs may provide genetic resistance to antibiotics and augment pathogenicity. ${ }^{80}$ Local application of $\mathrm{Zn}$ ointments enhances MTs, which induces cell proliferation and wound healing. ${ }^{81}$ Thus, material composition and design of fracture patties for amputees, wound clips, and dressings coated with $\mathrm{Ag}$ and $\mathrm{Au}$ NPs are being developed to prevent osteomyelitis, septicemia, inflammation, and allergic reactions. ${ }^{82}$ In addition, drug (sirolimus)-eluting carbon nanocoated stents are being developed to prevent platelet activation/aggregation, 5-hydroxytryptamine release, vascular neointimal hyperplasia, restenosis, thrombosis, and persistent infections due to the formation of biofilms on these nanodevices. ${ }^{83}$ By altering the surface topography of nanocoatings, the activation of platelets can be affected, while the carbon nanocoatings having higher surface roughness is less thrombogenic in terms of platelet adhesion for improving the stent coating fabrication. ${ }^{84}$ MT-capped $\mathrm{Cd} / \mathrm{Se}$ NPs are being further developed for their clinical application as theranostic agents. ${ }^{59}$ Further studies in this direction will go a long way in the effective clinical management of patients.

\section{Conclusion}

MTs have a diversified and versatile therapeutic role as free radical scavengers in the CNS and other tissues. Through $\mathrm{Zn}$-mediated transcriptional activation, these proteins can regulate cellular growth and development in health and disease. MTs induction in response to nutritional stress and environmental toxicity (particularly from glutamate analogs and NPs) is of considerable clinical interest. Hence it is 
important to verify NPs-mediated MTs induction before they could be used routinely as potential theranostic agents. As NPs aggregate and accumulate quite frequently in the biological system, it would of significant interest to study the contribution of MT induction and CB formation in these conditions. Furthermore, liposomal encapsulation and MT induction in stem cell therapeutics will have a promising future for developing safe and effective NPs for biomedical applications.

\section{Acknowledgments}

Moral support and encouragement by Dr Kallol Guha, Saint James School of Medicine, Bonaire is gratefully acknowledged.

\section{Disclosure}

The authors report no conflicts of interest in this work.

\section{References}

1. Marghoshes M, Vallee BL. A cadmium protein from equine kidney cortex. J Am Chem Soc. 1957;79:4813-4814.

2. Itoh M, Ebadi M, Swanson S. The presence of zinc-binding proteins in brain. J Neurochem. 1983;41(3):823-829.

3. Rupp $\mathrm{H}$, Weser U. Conversion of metallothionein into $\mathrm{Cu}$-thionein, the possible low molecular weight form of neonatal hepatic mitochondrocuprein. FEBS Lett. 1974;44(3):293-297.

4. Vasak M, Galdes A, Hill HA, Kagi JH, Bremner I, Young BW. Investigation of the structure of metallothioneins by proton nuclear magnetic resonance spectroscopy. Biochemistry. 1980;19(3):416-425.

5. Vasak M, Kagi JH, Hill HA. Zinc(II), cadmium(II), and mercury(II) thiolate transitions in metallothionein. Biochemistry. 1981;20(10): 2852-2856

6. Boulanger Y, Goodman CM, Forte CP, Fesik SW, Armitage IM. Model for mammalian metallothionein structure. Proc Natl Acad Sci U S A. 1983;80(6):1501-1505.

7. Braun W, Vasak M, Robbins AH, et al. Comparison of the NMR solution structure and the X-ray crystal structure of rat metallothionein-2. Proc Natl Acad of Sci U S A. 1992;89(21):10124-10128.

8. Zangger K, Oz G, Otvos JD, Armitage IM. Three-dimensional solution structure of mouse (Cd7)-metallothionein-1 by homonuclear and heteronuclear NMR spectroscopy. Protein Sci. 1999;8(12):2630-2638.

9. Nielsen AE, Bohr A, Penkowa M. The balance between life and death of a cell: role of metallothioneins. Biomark Insights. 2007;1: 99-111.

10. Pastore A, Piemonte F. S-glutathionylation signaling in cell biology: progress and prospects. Eur J Pharm Sci. 2012;46(5):279-292.

11. Khatai L, Goessler W, Lorencova H, Zangger K. Modulation of nitric oxide-mediated metal release from metallothionein by the redox state of glutathione in vitro. Eur J Biochem. 2004;271(12):2408-2416.

12. Helal GK, Aleisa AM, Helal OK, et al. Metallothionein induction reduces caspase-3 activity and TNFalpha levels with preservation of cognitive function and intact hippocampal neurons in carmustine-treated rats. Oxid Med Cell Longev. 2009;2(1):26-35.

13. Min KS. Physiological significance of metallothionein in oxidative stress. Yakugaku Zasshi. 2007;127(4):695-702. Japanese [with English abstract].

14. Kooncumchoo P, Sharma S, Porter J, Govitrapong P, Ebadi M. Coenzyme $\mathrm{Q}_{10}$ provides neuroprotection in iron-induced apoptosis in dopaminergic neurons. J Mol Neurosci. 2006;28(2):125-141.
15. Nath R, Kambadur R, Gulati S, Paliwal VK, Sharma M. Molecular aspects, physiological function, and clinical significance of metallothioneins. Crit Rev Food Sci Nutr. 1988;27(1):41-85.

16. Uchida Y, Takio K, Titani K, Ihara Y, Tomonaga M. The growth inhibitory factor that is deficient in the Alzheimer's disease brain is a 68 amino acid metallothionein-like protein. Neuron. 1991;7(2):337-347.

17. Smith E, Drew PA, Tian ZQ, et al. Metallothionien 3 expression is frequently down-regulated in oesophageal squamous cell carcinoma by DNA methylation. Mol Cancer. 2005;4:42.

18. Sharma SK, Ebadi M. Metallothionein attenuates 3-morpoholinosydnonimine (SIN-1)-induced oxidative stress in dopaminergic neurons. Antioxid Redox Signal. 2003;5(3):251-264.

19. Ebadi M, Sharma SK. Peroxynitrite and mitochondrial dysfunction in the pathogenesis of Parkinson's disease. Antioxid Redox Signal. 2003;5(3):319-335.

20. Ebadi M, Sharma S. Metallothioneins 1 and 2 attenuate peroxynitriteinduced oxidative stress in Parkinson's disease. Exp Biol Med (Maywood). 2006;231(9):1576-1583.

21. Ebadi M, Brown-Borg H, El Refaey H, et al. Metallothionein-mediated neuroprotection in genetically engineered mouse models of Parkinson's disease. Brain Res Mol Brain Res. 2005;134(1):67-75.

22. Ebadi M, Sharma S, Wanpen S, Shavali S. Metallothionein isoforms attenuate peroxynityrite-induced oxidative stress in Parkinson's disease. In: Ebadi M, Pfeiffer RF, editors. Parkinson's Disease. Boca Raton, FL: CRC Press; 2004:739-765.

23. Ebadi M, Sharma SK, Muralikrishnan D, et al. MTs provides ubiquinone-mediated neuroprotection in Parkinson's disease. Proc West Pharmacol Soc. 2002;45:36-38.

24. Ebadi M, Sharma SK, Ghafourifar P, Brown-Borg H, El Refaey H. Peroxynitrite in the pathogenesis of Parkinson's disease and the neuroprotective role of metallothioneins. Methods Enzymol. 2005;396:276-298.

25. Sharma S, Ebadi M. Metallothioneins as early and sensitive biomarkers of redox signaling in neurodegenerative disorders. The IIOAB Journal. 2011;2(6):98-106.

26. Sharma S, Ebadi M. Therapeutic potential of metallothioneins as anti-inflammatory agents in polysubstance abuse. The IIOAB Journal. 2011;2(6):50-61

27. Lee SJ, Koh JY. Roles of zinc and metallothionein-3 in oxidative stressinduced lysosomal dysfunction, cell death, and autophagy in neurons and astrocytes. Mol Brain. 2010;3(1):30.

28. Sonn K, Pankratova S, Korshunova I, et al. A metallothionein mimetic peptide protects neurons against kainic acid-induced excitotoxicity. J Neurosci Res. 2010;88(5):1074-1082.

29. Dakshinamurti K, Sharma SK, Sundaram M, Watanabe T. Hippocampal changes in developing postnatal mice following intrauterine exposure to domoic acid. J Neurosci. 1993;13(10):4486-4495.

30. Gill SS, Hou Y, Ghane T, Pulido OM. Regional susceptibility to domoic acid in primary astrocyte cells cultured from the brain stem and hippocampus. Mar Drugs. 2008;6(1):25-38.

31. Pulido OM. Domoic acid toxicologic pathology: a review. Mar Drugs. 2008;6(2):180-219.

32. Rue E, Bruland K. Domoic acid binds iron and copper: a possible role for the toxin produced by the marine diatom Pseudo-nitzschia. Marine Chemistry. 2001;76:127-134.

33. Pedersen DS, Fredericia PM, Pedersen MO, et al. Metallic gold slows disease progression, reduces cell death and induces astrogliosis while simultaneously increasing stem cell responses in an EAE rat model of multiple sclerosis. Histochem Cell Biol. 2012;138(5):787-802.

34. Zappia E, Casazza S, Pedemonte E, et al. Mesenchymal stem cells ameliorate experimental autoimmune encephalomyelitis inducing T-cell anergy. Blood. 2005;106(5):1755-1761.

35. Lanza C, Morando S, Voci A, et al. Neuroprotective mesenchymal stem cells are endowed with a potent antioxidant effect in vivo. J Neurochem. 2009;110(5):1674-1684.

36. Mickova A, Buzgo M, Benada O, et al. Core/shell nanofibers with embedded liposomes as a drug delivery system. Biomacromolecules. 2012;13(4):952-962. 
37. Chen XA, Zhang LJ, He ZJ, et al. Plasmid-encapsulated polyethylene glycol-grafted polyethylenimine nanoparticles for gene delivery into rat mesenchymal stem cells. Int J Nanomedicine. 2011;6:843-853.

38. Scott RC, Rosano JM, Ivanov Z, et al. Targeting VEGF-encapsulated immunoliposomes to MI heart improves vascularity and cardiac function. FASEB J. 2009;23(10):3361-3367.

39. Hofkens W, Grevers LC, Walgreen B, et al. Intravenously delivered glucocorticoid liposomes inhibit osteoclast activity and bone erosion in murine antigen-induced arthritis. J Control Release. 2011;152(3) 363-369.

40. Tang S, Liu Z, Zhao L, Zou Z, Du M. Transfection of pEGFP-C2 in brain mediated by targeting liposome P-MMA-DOSPER. Sheng $W u Y i$ Xue Gong Cheng Xue Za Zhi. 2008;25(5):1170-1174. Chinese.

41. Giuliani AL, Wiener E, Lee MJ, Brown IN, Berti G, Wickramasinghe SN. Changes in murine bone marrow macrophages and erythroid burstforming cells following the intravenous injection of liposomeencapsulated dichloromethylene diphosphonate (C12MDP). Eur $J$ Haematol. 2001;66(4):221-229.

42. Aksentijevich I, Pastan I, Lunardi-IskandarY, Gallo RC, Gottesman MM, Thierry AR. In vitro and in vivo liposome-mediated gene transfer leads to human MDR1 expression in mouse bone marrow progenitor cells. Hum Gene Ther. 1996;7(9):1111-1122.

43. Rahman YE, Hanson WR, Bharucha J, Ainsworth EJ, Jaroslow BN. Mechanisms of reduction of antitumor drug toxicity by liposome encapsulation. Ann N Y Acad Sci. 1978;308:325-342.

44. Wang Y, Zeng B, Li X. Expression of human calcitonin by microencapsulated recombinant myoblasts. Biotechnol Lett. 2006;28(18): 1453-1458.

45. Xie Y, Ye L, Zhang X, et al. Transport of nerve growth factor encapsulated into liposomes across the blood-brain barrier: in vitro and in vivo studies. J Control Release. 2005;105(1-2):106-119.

46. Hatakeyama H, Ito E, Yamamoto M, et al. A DNA microarraybased analysis of the host response to a nonviral gene carrier: a strategy for improving the immune response. Mol Ther. 2011;19(8) $1487-1498$.

47. Hatakeyama H, Akita H, Ito E, et al. Systemic delivery of siRNA to tumors using a lipid nanoparticle containing a tumor-specific cleavable PEG-lipid. Biomaterials. 2011;32(18):4306-4316.

48. Hatakeyama $\mathrm{H}$, Akita $\mathrm{H}$, Harashima $\mathrm{H}$. A multifunctional envelope type nano device (MEND) for gene delivery to tumours based on the EPR effect: a strategy for overcoming the PEG dilemma. Adv Drug Deliv Rev. 2011;63(3):152-160.

49. Kawaguchi AT, Kametani Y, Kato S, Furuya H, Tamaoki K, Habu S. Effects of liposome-encapsulated hemoglobin on human immune system: evaluation in immunodeficient mice reconstituted with human cord blood stem cells. Artif Organs. 2009;33(2): 169-176.

50. Wong A. Modified epidermal growth factor receptor (EGFR)-bearing liposomes (MRBLs) are sensitive to EGF in solution. PLoS One. 2009;4(10):e7391.

51. Xiao Q, Xiao C. Preparation and characterization of silica-coated magnetic-fluorescent bifunctional microspheres. Nanoscale Res Lett 2009;4(9):1078-1084.

52. Leung K. Quantum dot-prostate-specific membrane antigen antibody J591. In: Molecular Imaging and Contrast Agent Database (MICAD). Bethesda, MD: National Center for Biotechnology Information; 2005. Available from: http://www.ncbi.nlm.nih.gov/books/NBK23039/pdf/ QD-PMSA.pdf. Accessed February 20, 2013.

53. Choi Y, Kim K, Hong S, Kim H, Kwon YJ, Song R. Intracellular protein target detection by quantum dots optimized for live cell imaging. Bioconjug Chem. 2011;22(8):1576-1586.

54. Lin CH, Chang LW, Chang H, et al. The chemical fate of the $\mathrm{Cd} / \mathrm{Se} / \mathrm{Te}-$ based quantum dot 705 in the biological system: toxicity implications. Nanotechnology. 2009;20(21):215101.

55. Chang CC, Sun KW, Lee SF, Kan LS. Self-assembled molecular magnets on patterned silicon substrates: bridging bio-molecules with nanoelectronics. Biomaterials. 2007;28(11):1941-1947.
56. Peyrot C, Gagnon C, Gagne F, Willkinson KJ, Turcotte P, Sauve S. Effects of cadmium telluride quantum dots on cadmium bioaccumulation and metallothionein production to the freshwater mussel, Elliptio complanata. Comp Biochem Physiol C Toxicol Pharmacol. 2009;150:246-251.

57. Wei L, Thakkar M, Chen Y, Ntim SA, Mitra S, Zhang X. Cytotoxicity effects of water dispersible oxidized multiwalled carbon nanotubes on marine alga, Dunaliella tertiolecta. Aquat Toxicol. 2010;100(2): 194-201.

58. Mounicou S, Ouerdane L, L'Azou B, et al. Identification of metallothionein subisoforms in HPLC using accurate mass and online sequencing by electrospray hybrid linear ion trap-orbital ion trap mass spectrometry. Anal Chemistry. 2010;82(16):6947-6957.

59. Neupane KP. Developing Metallothionein Capped Cadmium Selenide Nanoparticles [doctoral thesis]. Detroit, MI: Wayne State University; 2005.

60. Brenneman M, Sharma S, Harting M, et al. Autologous bone marrow mononuclear cells enhance recovery after acute ischemic stroke in young and middle-aged rats. J Cereb Blood Flow Metab. 2010;30(1):140-149.

61. Yang B, Strong R, Sharma S, et al. Therapeutic time window and dose response of autologous bone marrow mononuclear cells for ischemic stroke. J Neurosci Res. 2011;89(6):833-839.

62. Misra V, Yang B, Sharma S, Savitz S. Cell-based therapy for stroke. In: Cox CS, editor. Progenitor Cell Therapy for Neurological Injury. New York, NY: Humana Press; 2011:143-162.

63. Sharma S, Yang B, Strong R, et al. Bone marrow mononuclear cells protect neurons and modulate microglia in cell culture models of ischemic stroke. J Neurosci Res. 2010;88(13):2869-2876.

64. Sharma S, Yang B, Xi X, Grotta J, Aronowski J, Savitz SI. IL-10 directly protects cortical neurons by activating PI-3 kinase and STAT-3 pathways. Brain Res. 2011;1373:189-194.

65. El Khoury R, Misra V, Sharma S, et al. The effect of transcatheter injections on viability and cytokine release of mononuclear cells. AJNR Am J Neuroradiol. 2010;31(8):1488-1492.

66. Sharma S, Ebadi M. In vivo molecular imaging in Parkinson's disease In: Pfeiffer RF, Wszolek ZK, Ebadi M, editors. Parkinson's Disease, 2nd ed. Boca Raton, FL: CRC Press; 2013:787-802.

67. Liu Z, Ren G, Zhang T, Yang Z. Action potential changes associated with inhibitory effects on voltage-gated sodium currents of hippocampal CA1 neurons by silver nanoparticles. Toxicology. 2009;246(3):179-184.

68. Haase A, Rott S, Mantion A, et al. Effects of silver nanoparticles on primary mixed neural cell cultures: uptake, oxidative stress and acute calcium responses. Toxicol Sci. 2012;126(2):457-468.

69. Luther EM, Schmidt MM, Diendorf J, Epple M, Dringen R. Upregulation of metallothioneins after exposure of cultured primary astrocytes to silver nanoparticles. Neurochem Res. 2012;37(8):1639-1648.

70. Pan JF, Buffet PE, Poirier L, et al. Size dependent bioaccumulation and ecotoxicity of gold nanoparticles in an endobenthic invertebrate: the Tellinid clam Scrobicularia plana. Environ Pollut. 2012;168:37-43.

71. Pan Y, Leifert A, Graf M, et al. High-sensitivity real-time analysis of nanoparticle toxicity in green fluorescent protein-expressing zebrafish. Small. Epub 2012 Nov 12.

72. Fan J, Gallagher JW, Wu HJ, et al. Low molecular weight protein enrichment on mesoporous silica thin films for biomarker discovery. J Vis Exp. 2012;(62):3876.

73. Steenland K, Stayner L. Silica, asbestos, man-made mineral fibers, and cancer. Cancer Causes Control. 1997;8(3):491-503.

74. Bertoni-Freddari C, Fattoretti P, Casoli T, Di Stefano G, Giorgetti B, Balietti M. Brain aging: the zinc connection. Exp Gerontol. 2008;43(5): 389-393.

75. Mocchegiani E, Costarelli L, Giacconi R, Piacenza F, Basso A, Malavolta M. Micronutrient ( $\mathrm{Zn}, \mathrm{Cu}, \mathrm{Fe})$-gene interactions in ageing and inflammatory age-related diseases: implications for treatments. Ageing Res Rev. 2012;11(2):297-319.

76. Derfus AM, Chan WCW, Bhatia SN. Probing the cytotoxicity of semiconductor quantum dots. Nano Lett. 2004;4(1):11-18.

77. Leigh K, Bouldin J, Buchanan R. Effects of exposure to semiconductor nanoparticles on aquatic organisms. J Toxicol. 2012;2012:397657. 
78. Penkowa M. Metallothioneins are multipurpose neuroprotectants during brain pathology. FEBS J. 2006;273(9):1857-1870.

79. Penkowa M, Tio L, Giralt M, et al. Specificity and divergence in the neurobiologic effects of different metallothioneins after brain injury. J Neurosci Res. 2006;83(6):974-984.

80. Stankovic RK, Chung RS, Penkowa M. Metallothioneins I and II: neuroprotective significance during CNS pathology. Int J Biochem Cell Biol. 2007;39(3):484-489.

81. Lansdown AB, Mirastschijski U, Stubbs N, Scanlon E, Agren MS. Zinc in wound healing: theoretical, experimental, and clinical aspects. Wound Repair Regen. 2007;15(1):2-16.
82. Wong KK, Cheung SO, Huang L, et al. Further evidence of the anti-inflammatory effects of silver nanoparticles. Chem Med Chem. 2009;4(7):1129-1135.

83. Rajender G, Narayanan NG. Liquid chromatography-tandem mass spectrometry method for determination of sirolimus coated drug eluting nano porous carbon stents. Biomed Chromatogr. 2010;24(3): 329-334.

84. Karagkiozaki VC, Logothetidis SD, Kassavetis SN, Giannoglou GD. Nanomedicine for the reduction of the thrombogenicity of stent coatings. Int J Nanomedicine. 2010;5:239-248.

\section{Publish your work in this journal}

The International Journal of Nanomedicine is an international, peerreviewed journal focusing on the application of nanotechnology in diagnostics, therapeutics, and drug delivery systems throughout the biomedical field. This journal is indexed on PubMed Central, MedLine, CAS, SciSearch ${ }^{\circledR}$, Current Contents ${ }^{\circledR} /$ Clinical Medicine,
Journal Citation Reports/Science Edition, EMBase, Scopus and the Elsevier Bibliographic databases. The manuscript management system is completely online and includes a very quick and fair peer-review system, which is all easy to use. Visit http://www.dovepress.com/ testimonials.php to read real quotes from published authors. 